Acta Cryst. (1992). A48, 785-790

\title{
International Union of Crystallography Keywords for the Database of Crystallographers and the World Directory
}

\author{
Y. Epelboin, General Editor, LMCP, Universités P. M. Curie et Paris VII, URA 009,
} CNRS, Case 115, 75252 Paris CEDEX 05, France [e-mail: epelboin@lmcp.jussieu.fr]

The International Union of Crystallography will set up a world database of crystallographers. The next issue of the World Directory will be a by-product of this database.

The aim is to allow any scientist to retrieve useful information on other scientists: addresses, interests ... . The database will be accessed by e-mail and later via telnet sessions. Security will be enforced to ensure that the data are not used for non-scientific purposes.

One of the main uses is to find specialists on given topics. This means that it will be possible to search the database by keywords and these must be defined in advance. The present list has been established on the basis of the keywords used in the eighth edition of the World Directory. Some additional keywords corresponding to new fields have been added. Some, too specific or misspelled, have been suppressed. Altogether there are about 1500 keywords.
Scientists will be able to use their own keywords for a better definition of their fields of interest but electronic searching of the database will be based on this printed list.

The collection of data will start at the beginning of 1993 and instructions will be distributed by the national Sub-Editors.

The list is divided into three parts: - Methods, Properties and Applications - Compounds - Attributes. The Attributes list must be used in conjunction with the other two lists and defines additional keywords for a better description of entries in those lists.

I hope that everybody will find appropriate definitions in the present lists. For maximum efficiency of the search process it is necessary to bear in mind that a too strict definition will be useless. This is one of the key points for the success of this database.

\section{Methods, Properties and Applications}

This list contains the keywords for methods of study, properties (physical, chemical, biological, ... ) and applications. It may be used with words defined in the Attributes list.

Aberration

Absolute configuration

Absolute structure

Absorption correction

Absorption edge

Absorption spectroscopy

Accuracy

Accurate intensity

Acoustics

Acoustooptics

Activity

Adhesion

Adrenergics

Adsorbate

Adsorption

AEM

Aerodynamics

Aerosol

Aerospace

AES

Affinity

Ageing process

Agriculture

Algorithm

ALISUVAX

Allostery

Alteration

Amorphization

Amorphous phase

Analgesics

Anharmonicity

Anisotropy

Annealing

Anomalous dispersion

Anomeric effect

Antiferroelectricity

Antiferromagnetism
Antiphases

Anvil cell

Aperiodic material

Apparatus

Archeology

Archeometallurgy

Archeometry

Area detector

Art conservation

Arthropatic disease

Artificial intelligence

ARUPS

ASAXS

Association theory

Astronomy

Astrophysics

Asymmetric synthesis

Asymmetry

Athletic medicine

Atomic weight

Attenuation coefficient

Auger spectroscopy

Automation

Autometasomatism

Back-reflection

Ballistic

Band calculation

Basicity relationship

Battery

Bijvoet absorption edge

Biochemistry

Biocoordination

Biocrystallography

Bioelectret

Bioenergetics

Biology

Biomaterial
Biomechanics
Biomolecule
Biophysics
Biosynthesis
Birefringence
Bloch structure
Bloch wall
Bond length
Bond method
Bond order
Bonding
Born approximation
Borrmann absorption
Boundaries
Bragg intensity
Bravais lattice
Bridgman Stockbarger
technique
Brillouin spectroscopy
Burial diagenesis
Calcification
Calibration
Calorimetry
Camera
Carboxylation
Carcinogenesis
Catalysis
Centrosymmetry
Chandler wobble
Channelling
Characterization
Charge density
Charge-density wave
Charge localization
Charge transfer

Chelation

Chemisorption

Chemistry

Chemometrics

Chemotaxis

Chemotherapy

Chirality

Chromatography

Circular dichroism

Classification

Clinker

Close packing

Cloud physics

Clustering

Coagulation

Coalification

Coarsening

Coating

Codification

Cohesion

Cohesive energy

Colour center

Colour symmetry

Combinatorial theory

Combustion

Complexation

Compliance sampling

Compression

Compton scattering

Computer

Computer-aided education

Computer architecture

Computer-assisted design

Computer automation

Computer graphics

Computer management

Computer modelling
Computer sciences

Computer technology

Computing

Condensed matter

Conductivity

Conductor

Conformation

Conformational change

Contaminant clean-up

Contractile system

Contrast

Control

Convective heat

Convergent-beam diffraction

Cooperative interaction

Cooperative phenomena

Coordination

Corrosion

Cosmochemistry

Crack

Cracking

Creep

Critical phenomena

Cross section

Cryogenics

Crystal field

Crystal force

Crystal form

Crystal growth

Crystallinity

Crystallite

Crystallization

Crystallogeny

Crystallography

CVD

Cycloaddition

Czochralski technique 


\section{Damage}

Data collection

Data processing

Database

Debye Scherrer

Debye temperature

Deby Waller factor

Decay

Decomposition

Deconvolution

Decoration

Defect

Deformation

Densitometry

Density distribution

Depolarization

Deposition

Derivative structure

Design

Desmearing

Detector

Devitrification

Diagnostic

Dielectrics

Differential thermal analysis

Diffraction

Diffraction data

Diffraction technique

Diffraction theory

Diffractometer

Diffractometry

Diffuse scattering

Diffusion

Digital signal processing

Diode

Direct method

Dirichlet domain

Discrete mathematics

Dislocation

Disorder

Dispersion

Dispersive system

Displacive modulation

Dissolution

Distribution functions

Divergent-beam method

Documentation

Domain structure

Dosimetry

Doublet separation

Drug

DSC

DTA

Dynamical diffraction

Dynamical property

Dynamics

Economy

Edaphology

EDAX

EDS

Education

EDX

EELS

EF hand

Elasticity

Electrochemistry

Electrocrystallization

Electrodeposition

Electrofusion

Electrolysis
Electroluminescence

Electromechanics

Electron beam

Electron microscope tomography

Electron microscopy

Electron probe microanalysis

Electronics

Electronic spectrum

Electron spin resonance

Electrooptics

Electrophotography

Electroporation

Electrostatic potential

Electrostatics

Embrittlement

Endocrinology

Energetics

Energy

Energy band

Energy conversion

Energy-dispersive analysis

Energy loss

Energy transduction

Engineering

Entropy

Environment

Environmental cracking

Environment protection

Epitaxy

EPR

ESCA

Etching

EXAFS

Exciton

Expert system

Exsolution

Extinction

Extraction

Extremal condition

Failure analysis

Fankuchen effect

Fast chemical reaction

Fatigue

Fault

Fermion

Ferroelasticity

Ferroelectricity

Field calculation

Field displacement

Field ion microscopy

Field ionization

Field theory

Film

Filter

FIR

Fission product

Fitting

Fixation

Flash X-ray diffraction

Float zone growth

Flotation

Fluorescence

Flux

Focused ion beam

Folding

Forbidden reflection

Force

Force constant
Force field

Form

Formability

Form factor

Four-dimensional crystallography

Fourier transform

Fractal

Fracture

Framework structure

Free energy

FTIR

Fuel cell

Function

Funding

Furnace

Gamma ray

Gap junction

Gasification

Gemology

Genesis

Geochemistry

Geochronology

Geodynamics

Geology

Geomagnetism

Geometry

Geometry analysis

Geomorphology

Geophysics

Geosciences

Geotechnics

Germination

Gerontology

GIXS

Glaciology

Goniometry

Graphics

Graph theory

Grazing incidence

Group theory

Growth

Hall effect

Hardening

Hardness

Heat transfer

Heavy atom

Heavy fermion

Heterophase

Heterostructure

High energy

High-energy electron diffraction

High-energy electron microscopy

High field

High-precision diffractometry

High-precision structures

High pressure

High purity

High-resolution diffractom-

etry

High-resolution electron mi-

croscopy

High temperature

High voltage

Histocompatibility

History

Hole centre
Holography

Homology

Homophase

Hydration

Hydrodynamics

Hydroelectrodynamics

Hydrogen bonding

Hydrolysis

Hydrotreating

Ideal structure

Identification

Image processing

Image reconstruction

Imaging

Immune regulation

Immunoassay

Immunobiology

Immunochemistry

Immunodeficiency

Immunology

Imperfection

Implantation

Impurity

Inclusion

Incoherent scattering

Incommensurate

Indexing

Industry

Inelastic scattering

Information science

Information storage

Information system

Infrared

Infrared detector

Inhibition

Inhibitor

Instability

Instrumentation

Integrated circuit

Integrated optics

Intensity

Interaction

Interatomic distance

Intercalation

Interface

Interference

Interferometry

Intermediate phase

Internal friction

International Tables for Crystallography

Inverse problem

Ion beam

Ion exchanger

Ionic conductivity

Ion implantation

Irradiation

Isomorphism

Isomorphous replacement

Jahn Teller effect

Kikuchi effect

Kinetics

Kohn anomaly

Kossel diffraction

Langmuir Blodgett film

Langmuir monolayer

Laser

Lattice distortion

Lattice dynamics

Lattice energy

Lattice parameter

Lattice stability

Lattice vibration

Laue diffraction

Laue group

Law

Layer 
Microgravity

Microlithography

Micromagnetism

Micrometre scale

Micromethod

Micromorphology

Microprocessor

Microscopy

Microstrain

Microstructure

Microtexture

Microtomography

Microwave

Mineralization

Mineralogy

Minimization

Mining

Mirrors

Miscibility

Misorientation

Mobility

MO calculation

Modelling

Modulated structures

Moire

Molecular beam

Molecular crystal

Molecular mechanics

Molecular rectifier

Molecular replacement

Molecular vibration

Momentum density

Momentum distribution

Monitoring

Monochromator

Monocrystal

Monolayer

Monte Carlo

Morphology

MOS

Mosaicity

Mossbauer

Motion

Multibeam

Multicrystal

Multidomain

Multilayer

Multiphase

Multiple-crystal diffractometry

Multiple scattering

Multislice method

Mutagenesis

Mycology

Nanoanalysis

Neurochemistry

Non-destructive analysis

Non equilibrium

Non-linear property

Nonstoichiometry

NPR

NQR

Nuclear filter

Nuclear fusion

Nuclear magnetic resonance

Nuclear reactor

Nucleation

Number theory

Nutrition

Occupancy
OD

Oncology

One dimension

Ontogeny

Optical activity

Optical property

Optical transform

Optics

Optimization

Optoelectrical property

Optoelectronics

Orbital calculation

Order

Order-disorder

Ordered structure

Ordering

Orientation

Orogenic belt

Oscillation camera

Ostwald ripening

Overcrowding

Oxidation

Packing

Paleomagnetism

Paracrystal

Paragenesis

Paramagnetic resonance

Paramagnetics

Parameter

Patent

Pattern recognition

Patterson method

Perfect crystal

Perfection

Performance

Pericyclic reaction

Permittivity

Petrography

Petrology

Pharmacology

Phase determination

Phase diagram

Phase equilibrium

Phase formation

Phase kinetics

Phase refinement

Phase separation

Phase transition

Philosophy

Philosophy of science

Phonon resonance

Phonon softening

Photochemistry

Photochromism

Photoconductivity

Photodimerization

Photoelasticity

Photoelectron

Photoemission

Photogeology

Photography

Photon effect

Photoreaction centre

Photorearrangement

Photorefraction

Photostimulated process

Photosynthesis

Phylogeny

Physical property

Physics
Physiology

Pi electron

Piezoelectricity

Pigment

Pitch

Planar defect

Planetology

Planning

Plasmon

Plastic flow

Plasticity

Plastics

Platelet

Point defect

Point group

Poisoning

Polarity

Polarization

Polarization microscopy

Polarized neutron

Pole figure

Pollution

Polycrystal

Polymerization

Polymorphism

Polytypism

Porosity

Positron annihilation

Potential energy

Powder

Precession

Precipitation

Precise measurement

Prediction

Preparation

Pressure

Processing

Profile analysis

Proportional counter

Prosthesis

Pseudomorphism

Pseudosymmetry

Publishing

Pulsed neutron

Purification

PVD

Pyroelectricity

QSAR

Quadrupole resonance

Qualitative analysis

Quantum mechanics

Quasicrystal

Radiation

Radiation protection

Radioactivity

Radiochemistry

Radiotracer

Raman

Random phasing method

Random system

Random walk

Rayleigh scattering

Reactivity

Real crystal

Real structure

Real-time control

Real-time imaging

Rearrangement

Receptor

Recognition
Recombination

Reconstruction

Recrystallization

Refinement method

Reflectance

Reflected light microscopy

Reflectivity

Refractive index

Regulation

Relaxation

Reliability

REM

Remote control

Repair

Replacement

Replication

Representation theory

Research

Residual electron density

Residual stress

Resistivity

Resonance

Resonance spectrometry

Resonant scattering

Restrained least squares

Reversible reaction

RHEED

Rietveld method

Rigid-body analysis

Risk assessment

Rocking curves

Rotatory dispersion

Safety

Satellite reflection

SAXS

Scale factor

Scale mechanism

Scanning electron microscopy

Scanning tunnel microscopy

Scattering

Scattering factor

Sciences

Search and match

Secondary bonding

Secondary electron emission

Sedimentation

Seismology

Selectivity

Semiconductor

Semi-empirical calculation

Sensor

Sequencing

Service

Shape

Shape memory

Shock metamorphism

Shock wave

Short hydrogen bond

Short-range order

SIMS

Simulation

Simultaneous diffraction

Single crystal

Sintering

Size distribution

Size effect

Slow neutron

Small-angle scattering

Soft mode

Software

Soft X-ray

Solar cell

Solar collector

Solar energy

Solidification

Solid phase

Solid solution 
Symmetry

Symmetry breaking

Symmetry group

Synchrotron radiation

Syncrystallization

Synthesis

Systematics

System dynamics

System integration

Tautomerism

Technique

Technology

Television

Temperature

Tensor

Tensometry

Tensor property

Termination effect

Tertiary structure

Testing

\begin{tabular}{|c|c|}
\hline Texture & Topochemistry \\
\hline TGA & Topography \\
\hline Theory & Topology \\
\hline Thermal expansion & Topotacticity \\
\hline Thermal motion & Topotaxy \\
\hline Thermal property & Toxicity \\
\hline Thermal stress & Toxicology \\
\hline Thermal vibration & Trace \\
\hline Thermistor & Trace analysis \\
\hline Thermoanalysis & Track detector \\
\hline Thermodynamics & Transcription \\
\hline Thermogravimetry & Transducer \\
\hline Thermoluminescence & Transduction \\
\hline Thermostability & Transformation \\
\hline Thick film & Transmission electron \\
\hline Thin film & microscopy \\
\hline Thin layer & Tribology \\
\hline Three-dimensional & Triplet \\
\hline reconstruction & Tube \\
\hline Time-resolved effect & Tunnelling \\
\hline Time-of-flight diffraction & Twin \\
\hline
\end{tabular}

Texture

Theory

Thermal expansion

Thermal stress

Thermal vibration

Thermisto

Thermogravimetry

Thermoluminescence

Thin film

Thin laye

reconstruction

Time-of-flight diffraction

Twinning
Typomorphism
Ultra high pressure
Ultra high vacuum
Ultra pure compound
Ultrasonics
Ultraviolet
Unit cell
Unusual bonding
UPS
Vacancy
Vacuum
Valence charge density
van der Waals radius
Vector search
Vibration
Vitreous state
Volatility
Volcanology
VVPES

Wavelength

WAXS

WDS

Weak-beam electron diffraction

Weak interaction

Weathering

Welding

Whisker

White-beam radiation

Wide-angle scattering

Wigner crystal

XANES

XPS

$\mathrm{X}$-ray fluorescence

$\mathrm{X}$-ray fluorescence spectroscopy

Yeast expression system

Ylides

\section{Compounds}

This list contains classes of compounds and more general names to define classes of materials, such as Magnets. It may be used with words defined in the Attributes list.

\begin{tabular}{|c|c|c|c|c|}
\hline Acetylene & Antigens & Basaltic rock & Catalysts & Dichalcogenides \\
\hline Acids & Antihistaminic compounds & Bases & Celluloses & Dielectrics \\
\hline Actin & Antihypertensive com- & Bauxite & Cements & Dihydrofolate \\
\hline Actinides & pounds & Beryl & Ceramics & Dipeptide \\
\hline Adenoviruses & Anti-inflammatory com- & Beryllium compounds & Chalcogenides & Disease \\
\hline Adrenergic compounds & pounds & Bile pigments & Chalcogens & Dismutases \\
\hline Aggregates & Anti-influenza compounds & Bimetallic compounds & Chalcopyrites & Diterpenes \\
\hline AIDS & Antileprosy compounds & Binary alloys & Chelates & Diuretics \\
\hline Air & Antileukemia compounds & Bioceramics & Chlorine compounds & DNA \\
\hline Albumin & Antimalarial compounds & Biopolymers & Chlorites & Drug \\
\hline Alkaline & Antimicrobial compounds & Bismuth compounds & Chromatin & Dust \\
\hline Alkalis & Antimitotic compounds & Blende & Chromite & Dyes \\
\hline Alkaloids & Antimony compounds & Blood & Chromium compounds & Elastomers \\
\hline Alkanes & Antimuscarinic compounds & Boehmite & Chrysotile & Electroceramics \\
\hline Alkoxides & Antioxidants & Bone & Clathrates & Electrolytes \\
\hline Allergenics & Antiparasitic compounds & Boron compounds & Clays & Energetic compounds \\
\hline Alloys & Anti-Parkinsonian com- & Borophosphates & Clusters & Enkephalins \\
\hline Alumina & pounds & Borosilicates & Coal & Enzyme inhibitors \\
\hline Aluminate & Antipsychotic compounds & Bromium compounds & Cobalt compounds & Enzymes \\
\hline Aluminium compounds & Antipyretics & Bronzes & Coke & Estrogens \\
\hline Aluminosilicates & Antirheumatic compounds & Buffers & Colloids & Expectorants \\
\hline $\begin{array}{l}\text { Amino acids } \\
\text { Analgesics }\end{array}$ & Antischistosomal com- & Bushveld complex & Conglomerates & Explosives \\
\hline Anorthosite & $\begin{array}{l}\text { pounds } \\
\text { Antischizophrenia com- }\end{array}$ & Cadmium compounds & $\begin{array}{l}\text { Copper compounds } \\
\text { Cordierite }\end{array}$ & Fab fragments \\
\hline Antiallergenics & pounds & Cage molecules & Crown compounds & Fats \\
\hline Antiamoebic compounds & Antisickling compounds & Calcium compounds & Crust & Feldspars \\
\hline Antiangional compounds & Antispasmodics & $\begin{array}{l}\text { Cancer } \\
\text { Carbanions }\end{array}$ & Cryptates & Ferrites \\
\hline Antiarrhythmic compounds & Antithrombotic compounds & $\begin{array}{l}\text { Carbanions } \\
\text { Carbides }\end{array}$ & Cubanes & Fertilizers \\
\hline Antiarthritic compounds & Antitumour compounds & Carbohydrates & Cyanide & Fibres \\
\hline Antiasthmatic compounds & Antiulcer compounds & Carbonates & Cyanins & Fire-resistant compounds \\
\hline Antibacterial compounds & Antiviral compounds & Carbonyls & Cyclic polyethers & Flavonoids \\
\hline Antibiotics & Anxiolitic compounds & Carbon compounds & Cyclodextrins & Fluids \\
\hline Antibodies & Apatite & Carboranes & Cyclophosphazenes & Fluoride \\
\hline Anticancer compounds & Archeological materials & Carboxylates & Cytochrome & Fluorine compounds \\
\hline Anticholinergic compounds & Arsenic compounds & Carboxylic acids & Cytoplasm & Fluorometallates \\
\hline Anticoagulants & Asbestos & Carboxypeptidases & Cytotoxins & Fluoroorganics \\
\hline Anticonvulsants & Austenite & Carcinogens & Dehydrogenases & Fossils \\
\hline Antidepressants & Bacterial compounds & Carcinostats & Dental material & $\begin{array}{l}\text { Free radicals } \\
\text { Fuel }\end{array}$ \\
\hline Antiemetics & Bacterial toxin & Cardenolides & Detergents & Fulgides \\
\hline Antiestrogen compounds & Barbiturates & Cardiac compounds & Diamond & Fungicides \\
\hline $\begin{array}{l}\text { Antifolates } \\
\text { Antigelling compounds }\end{array}$ & Barium compounds & Cascade proteins & Diaspores & Fused rings \\
\hline
\end{tabular}




\section{Attributes}

This list contains additional keywords which may be used together with those defined in the Compounds and Methods, Properties and Applications lists.

\begin{tabular}{|c|c|c|c|c|}
\hline Absolute & Commensurate & Formation & Mass & Qualitative \\
\hline Absorbing & Comparison & Four-dimensional & Material & Quantitative \\
\hline Accurate & Complex & Gamma-ray & Mathematical & Quantum \\
\hline Acid & Composite & $\begin{array}{l}\text { Gamma-ray } \\
\text { Genetic }\end{array}$ & Mechanical & Quasielastic \\
\hline Acoustic & Composition & Geochemical & Medical & Quaternary \\
\hline Activation & Compound & Geometric & Medicinal & Rapid \\
\hline Active site & Condensed & Geothermal & Medium-size & Reaction \\
\hline Active surface & Conducting & Globular & Mesogenic & Refinement \\
\hline Acyclic & Conformational & Glycolytic & Metallic & Reflection \\
\hline Adduct & Constituent & Halophilic & Metalloorganic & Relationship \\
\hline Agrochemical & Cosmic & Heavy & Metallurgical & Relative \\
\hline Amorphous & Crystal & $\begin{array}{l}\text { Heavy } \\
\text { Helical }\end{array}$ & Metamorphic & Residue \\
\hline Amphibole & Crystalline & $\begin{array}{l}\text { Helical } \\
\text { Heterocyclic }\end{array}$ & Metastable & Resolution \\
\hline Amphiphilic & Cubic & $\begin{array}{l}\text { Heterocyclic } \\
\text { Heterogeneous }\end{array}$ & Method & Respiratory \\
\hline Analysis & Cyclic & $\begin{array}{l}\text { Heterogeneous } \\
\text { Hexagonal }\end{array}$ & Mineralized & Restrained \\
\hline Analytical & Density & $\begin{array}{l}\text { Hexagonal } \\
\text { High }\end{array}$ & Mixed & Rhombohedral \\
\hline Anharmonic & Dependence & High-precision & Model & Ring \\
\hline Anhydrous & Deposit & Holographic & Modulated & Rolled \\
\hline Anion & Dielectric & Homogeneous & Molecular & Secondary \\
\hline Anisotropic & Difference & Hydrothermal & $\begin{array}{l}\text { Monochromatic } \\
\text { Monoclinic }\end{array}$ & Separation \\
\hline Anomalous & Diffuse & Hydrous & Monoclinic & Sequence \\
\hline Anorthic & Disordered & Hygroscopic & $\begin{array}{l}\text { Monoclonal } \\
\text { Mosaic }\end{array}$ & Short-period \\
\hline $\begin{array}{l}\text { Antiferroelastic } \\
\text { Antiferroelectric }\end{array}$ & Displacive & Icosahedral & $\begin{array}{l}\text { Mosaic } \\
\text { Multiple }\end{array}$ & Site \\
\hline $\begin{array}{l}\text { Antiferroelectric } \\
\text { Antiferromagnetic }\end{array}$ & Domain & Ideal & & Size \\
\hline $\begin{array}{l}\text { Antiferromagnetic } \\
\text { Application }\end{array}$ & Donor & Incoherent & $\begin{array}{l}\text { Non-ideal } \\
\text { Nematic }\end{array}$ & Slag \\
\hline $\begin{array}{l}\text { Application } \\
\text { Applied }\end{array}$ & Doped & Inelastic & Nematic & Small \\
\hline $\begin{array}{l}\text { Applied } \\
\text { Aqueous }\end{array}$ & Double & Infrared & Neurological & Small-angle \\
\hline $\begin{array}{l}\text { Aqueous } \\
\text { Asymmetric }\end{array}$ & Drug & Inorganic & Neutron & Smectic \\
\hline $\begin{array}{l}\text { Asymmetric } \\
\text { Asymptotic }\end{array}$ & Dynamic & Interaction & Non- & Solid \\
\hline & Dynamical & Interatomic & $\begin{array}{l}\text { Non-bonded } \\
\text { Non-crystalline }\end{array}$ & Soluble \\
\hline $\begin{array}{l}\text { Atmospheric } \\
\text { Atom }\end{array}$ & Efflorescent & Intercrystalline & $\begin{array}{l}\text { Non-crystalline } \\
\text { Non-crystallographic }\end{array}$ & Spectral \\
\hline Atomic & Elastic & Interfacial & $\begin{array}{l}\text { Non-crystallographic } \\
\text { Non-linear }\end{array}$ & Stainless \\
\hline Behaviour & $\begin{array}{l}\text { Electrical } \\
\text { Electromagnetic }\end{array}$ & Intermetallic & Nuclear-one-dimensional & Static \\
\hline Binary & $\begin{array}{l}\text { Electromagnetic } \\
\text { Electron }\end{array}$ & $\begin{array}{l}\text { Intermolecular } \\
\text { Internal }\end{array}$ & Optical & $\begin{array}{l}\text { Stereographic } \\
\text { Strained }\end{array}$ \\
\hline Binding & $\begin{array}{l}\text { Electron } \\
\text { Electronic }\end{array}$ & $\begin{array}{l}\text { Internal } \\
\text { Interstitial }\end{array}$ & Organic & $\begin{array}{l}\text { Strained } \\
\text { Structural }\end{array}$ \\
\hline Bioactive & Electrooptic & & Organometallic & Substituent \\
\hline Biochemical & Electrostatic & $\begin{array}{l}\text { Intracrystalline } \\
\text { Intramolecular }\end{array}$ & Orthorhombic & Superionic \\
\hline $\begin{array}{l}\text { Biogenic } \\
\text { Bioinorganic }\end{array}$ & Elongation & Intrazeolitic & Pathological & Synthetic \\
\hline $\begin{array}{l}\text { Bioinorganic } \\
\text { Biological }\end{array}$ & $\begin{array}{l}\text { Emission } \\
\text { Energy }\end{array}$ & Inverse & Perfect & Tensile \\
\hline $\begin{array}{l}\text { Biological } \\
\text { Biomedical }\end{array}$ & $\begin{array}{l}\text { Energy } \\
\text { Energy-disnercive }\end{array}$ & Ion & Pharmaceutical & Ternary \\
\hline $\begin{array}{l}\text { Biomedical } \\
\text { Bioorganic }\end{array}$ & Energy-dispersive & Ionic & Phase & Tetragonal \\
\hline $\begin{array}{l}\text { Bioorganic } \\
\text { Bond }\end{array}$ & $\begin{array}{l}\text { Environmental } \\
\text { Enzymatic }\end{array}$ & Irradiated & Phonon & Theoretical \\
\hline $\begin{array}{l}\text { Bond } \\
\text { Boundary }\end{array}$ & $\begin{array}{l}\text { Enzymatic } \\
\text { Epitaxic }\end{array}$ & Isometric & Phosphoorganic & Thermal \\
\hline $\begin{array}{l}\text { Boundary } \\
\text { Bragg }\end{array}$ & $\begin{array}{l}\text { Epitaxic } \\
\text { Equilibrium }\end{array}$ & Laminated & Photochromic & Thermophile \\
\hline & $\begin{array}{l}\text { Equilibrium } \\
\text { Evolution }\end{array}$ & $\begin{array}{l}\text { Lamunated } \\
\text { Large-angle }\end{array}$ & Photon & Toxic \\
\hline $\begin{array}{l}\text { Bridged } \\
\text { Building }\end{array}$ & $\begin{array}{l}\text { Evolution } \\
\text { Exchange }\end{array}$ & $\begin{array}{l}\text { Laue } \\
\text { Lange }\end{array}$ & Photovoltaic & Transfer \\
\hline Bulk & $\begin{array}{l}\text { Exchange } \\
\text { Excitation }\end{array}$ & Layered & Physical & Transport \\
\hline Catalytic & $\begin{array}{l}\text { Excitation } \\
\text { Experimental }\end{array}$ & Light & Piezoelectric & Treatment \\
\hline Cation & Exploration & Linear & Plastic & Two-dimensional \\
\hline Chain & Extended & Liquid & Polar & Unidirectional \\
\hline $\begin{array}{l}\text { Channel } \\
\text { Charge }\end{array}$ & Ferroelastic & $\begin{array}{l}\text { Local } \\
\text { Long-period }\end{array}$ & Polychromatic & Unsaturated \\
\hline $\begin{array}{l}\text { Charge } \\
\text { Chemical }\end{array}$ & Ferroelectric & $\begin{array}{l}\text { Long-period } \\
\text { Low }\end{array}$ & $\begin{array}{l}\text { Polycyclic } \\
\text { Polymeric }\end{array}$ & Vacancy \\
\hline Chemical & Ferroic & $\begin{array}{l}\text { Low } \\
\text { Low-dimensional }\end{array}$ & $\begin{array}{l}\text { Polymeric } \\
\text { Polymorphic }\end{array}$ & Vibrating \\
\hline $\begin{array}{l}\text { Chiral } \\
\text { Chiroptical }\end{array}$ & Ferromagnetic & Low-dimensional & Polytypic & Viral \\
\hline $\begin{array}{l}\text { Chiroptical } \\
\text { Chromatic }\end{array}$ & Fibrillous & $\begin{array}{l}\text { Macrocyclic } \\
\text { Macromolecular }\end{array}$ & Porous & Volatile \\
\hline Chromatic & Fibrous & Macromolecular & Process & Volcanic \\
\hline Clinical & Five-dimensional & Magmatic & Property & Wet \\
\hline Close-packed & Focusing & Magnetic & Pulsed & $\mathrm{X}$-ray \\
\hline Coherent & Forensic & $\begin{array}{l}\text { Marine } \\
\text { Martensitic }\end{array}$ & Pyrroelectric & Zone \\
\hline
\end{tabular}


Gallium compounds

Gallstones

Gases

Gelatins

Gels

Gemstones

Genes

Germanates

Germanium compounds

Glasses

Glycogens

Glycoproteins

Glycosaminoglycans

Glycosides

Gold compounds

Grains

Granites

Graphites

Halides

Halogens

Hemes

Hemoglobins

Hemoproteins

Herbicides

Heterocycles

Heteropoly acids

Heusler alloys

Histamine agonists

Hormones

HSLA steels

Humic compounds

Hydrates

Hydrides

Hydrogen compounds

Hydroxides

Hypnotics

Ice

II-VI compounds

III-V compounds

Immunoglobulins

Immuno modulators

Immunosuppressants

Indium compounds

Inhibitors

Insecticides

Insulin

Intercalates

Interstitial compounds

Invar

Iodine compounds

Ionic conductors

lonophores

Iridium compounds

Iron compounds

Isomers

Isopolymetallates

Isotopes

IV-VI compounds
Jahn Teller compounds

Lamellar compounds

Lamprophyres

Lamp materials

Lanthanides

Layered compounds

Lead compounds

Ligands

Lipases

Lipids

Lipoproteins

Liquid crystals

Liquids

Lithium compounds

Living systems

Lubricants

Luminescent compounds

Lymphocytes

Lymphokines

Macrocycles

Macromolecules

Magnesium compounds

Magnets

Main-group compounds

Manganese compounds

Mantle

Martensites

Materials

Melts

Membranes

Mercury compounds

Metallacarboranes

Metalloenzymes

Metallophthalocyanines

Metalloporphyrins

Metalloproteins

Metals

Meteorites

Micas

Micelles

Microcrystallite compounds

Minerals

Mixed-layer compounds

Mixed-valence compounds

Modulated structures

Molecular complexes

Molecules

Molybdates

Molybdenum compounds

Moon rocks

Multilayers

Muscarinic compounds

Muscles

Mutagenic compounds

Narcotics

Natural products

Nematogenic compounds

Nervous system
Neuroleptics

Neuropeptides

Neurotoxins

Nickel compounds

Niobium compounds

Nitrates

Nitrides

Nitrogenases

Nitrogen compounds

Noble gases

Noble metals

Nuclear materials

Nucleic acids

Nucleoproteins

Nucleosides

Nucleotides

Oils

Oligomers

Oligonucleotides

Oligopeptides

Oligosaccharides

Oncogenes

Opiates

Ores

Osmium compounds

Oxides

Oxygenases

Oxygen compounds

Oxyhydrides

Palladium compounds

Paper

Parasites

Particles

Penicillins

Peptaibols

Peptides

Perovskites

Pesticides

Phosphatases

Phosphates

Phosphorus compounds

Phosphorylases

Photochromic compounds

Photoconductors

Phyllosilicates

Pigments

Plagioclases

Plants

Plasmas

Platinum compounds

Plutonium compounds

Polar compounds

Polyamides

Polyanions

Polydentates

Polyelectrolytes

Polyesters

Polyimidazoles

Polyiodides
Polymerases

Polymers

Polyolefins

Polyoxoanions

Polypeptides

Polyphosphides

Polyproteins

Polysaccharides

Polythionates

Porous materials

Porphyrins

Potassium compounds

Powders

Precipitates

Propellants

Prostaglandins

Proteases

Proteins

Proteinases

Protein kinases

Prothrombins

Psychoactive compounds

Radicals

Radical salts

Radiopharmaceutical compounds

Radium compounds

Rare-earth compounds

Reductases

Refractory compounds

Renins

Rhenium compounds

Rhodium compounds

Ribosomes

Ring molecules

RNA

Rock

Rubber

Ruthenium compounds

Saccharides

Salts

Sandwich compounds

Sapidants

Sediments

Selenium compounds

Semiconductors

Semicrystalline compounds

Serums

Sesquiterpenes

Siderophores

Silicates

Silicon compounds

Silver compounds

Small molecules

Soaps

Sodium compounds

Soils

Solids

Sols

Solvents

Steels

Steroids

Sterols

Strontium compounds

Sulfates

Sulfides

Sulfur compounds

Superalloys

Superconductors

Superoxides

Surfactants

Sweeteners

Tantalum compounds

Technetium compounds

Tellurides

Tellurium compounds

Terpenes

Textiles

Therapeutic compounds

Thermoelectric materials

Thorium compounds

Thyrotoxic compounds

Tin compounds

Tissues

Titanates

Titanium compounds

Tooth compounds

Toxins

Tranquillizers

Transcriptases

Transition elements

Trypanosomes

Trypsins

Tumours

Tungstates

Tungsten compounds

Unidirectional compounds

Unsaturated compounds

Uranides

Uranium compounds

Uricosuric compounds

Vanadium compounds

Vasodilators

Venums

Viruses

Vitamins

Waste

Water

Waxes

Ytterbium compounds

Zeolites

Zinc compounds

Zirconium compounds 